\title{
Pro-apoptotic effect of TRAIL-transfected endothelial progenitor cells on glioma cells
}

\author{
XIN DENG ${ }^{1}$, WEN ZHAO ${ }^{2,3}$, LAIJUN SONG ${ }^{1}$, WEI YING ${ }^{1}$ and XINBIN GUO ${ }^{1}$ \\ ${ }^{1}$ Department of Neuro-Interventional Radiology, The First Affiliated Hospital of Zhengzhou University; ${ }^{2}$ Key Laboratory of \\ Advanced Pharmaceutical Technology, Ministry of Education of China; ${ }^{3}$ Co-innovation Center of Henan for New Drug R \& D \\ and Preclinical Safety; School of Pharmaceutical Sciences, Zhengzhou University, Zhengzhou, Henan 450001, P.R. China
}

Received July 29, 2017; Accepted January 11, 2018

DOI: $10.3892 / \mathrm{ol} .2018 .7977$

\begin{abstract}
Glioma is one of the most common aggressive neuroepithelial malignant tumors in the central nervous system. It has a high recurrence rate and poor prognosis, primarily due to the fact that novel therapeutic agents cannot penetrate the blood-brain barrier (BBB). Endothelial progenitor cells (EPCs) have been reported to move across the BBB and access the tumor site. However, whether EPCs expressing the tumor necrosis factor-related apoptosis-inducing ligand (TRAIL) induce glioma cell apoptosis requires further investigation. In the present study, EPCs were transfected and stably expressed with TRAIL through lentiviral infection. The pro-apoptotic effect of these TRAIL-expressing EPCs on the SHG44 glioma cell line was investigated. The migration ability of TRAIL-expressing EPCs toward SHG44 cells through the Transwell culture system was investigated via a high-content screening assay. The apoptotic rate and the expression of cleaved caspase- 8 and -3 in addition to the cleaved poly(ADP-ribose) polymerase in SHG44 cells significantly increased in the TRAIL-overexpressing EPC treatment group compared with the controls. The increased apoptotic rate was reversed using a caspase inhibitor. The findings suggested that the TRAIL-expressing EPCs induced apoptosis in the SHG44 cells by activating the death receptor pathway, indicating that the TRAIL-expressing EPCs may be a useful strategy for glioma treatment.
\end{abstract}

\section{Introduction}

Glioma is the most common malignant tumor in the central nervous system (CNS). It occurs in any part of the CNS and exhibits highly aggressive and malignant behavior. The survival rates of glioma remain at a low level after the traditional

Correspondence to: Professor Xinbin Guo, Department of Neuro-Interventional Radiology, The First Affiliated Hospital of Zhengzhou University, 1 Jianshedong Road, Erqi, Zhengzhou, Henan 450001, P.R. China

E-mail: gxb3906080@sina.com

Key words: apoptosis, cancer therapy, glioma, EPCs, TRAIL treatment, including surgical resection, radiotherapy, and chemotherapy $(1,2)$. Tumor necrosis factor-related apoptosis-inducing ligand (TRAIL) has become one of the hotspots in the last several years (3). TRAIL has shown great anticancer activity by selectively binding to death receptor 4 (DR4) and death receptor 5 (DR5) and inducing tumor cell apoptosis, while healthy cells remain affected (3-6). However, blood-brain barrier (BBB), as a protective mechanism for CNS tumors, prevents TRAIL from reaching tumor sites $(7,8)$. TRAIL cannot be applied in CNS cancer therapy owing to its short half-life $(9,10)$. TRAIL was combined with stem cells to address these issues. Both neural stem cells (NSCs) and mesenchymal stem cells (MSCs) were engineered to express TRAIL and restrict glioma cells, showing bright prospects (11-15). However, SCs had some limitations, such as uncertain differentiation and difficulties in acquisition from adults.

Endothelial progenitor cells (EPCs) are the precursors of endothelial cells, with CD31 and CD34 as their specific surface markers. They were first isolated in 1997 from human peripheral blood $(16,17)$. EPCs are recruited in the neovascularization of tumor and involved in tumor vascular network $(18,19)$. Owing to these characteristics, EPCs have the potential to be involved in anti-tumor therapies. Most previous studies explored anti-angiogenic treatment by suppressing the mobilization and homing of EPCs to tumors (20). Increasing attention has been paid to the use of EPCs as a vector for treatment $(21,22)$. Recent studies have demonstrated that EPCs can move across the BBB from peripheral blood and participate in the angiogenesis of gliomas $(23,24)$. Compared with NSCs and MSCs, EPCs can be obtained from peripheral blood, which means that EPCs can be easily acquired from adults. This makes the autotransplantation of EPCs possible and avoids immunological rejection. Therefore, EPCs have been recognized as an ideal vector to deliver antineoplastic molecules to CNS tumors. However, whether the TRAIL-expressing EPCs home to glioma cells through the BBB and induce cell apoptosis needs further investigation.

In this study, EPCs engineered with a lentivirus encoding TRAIL were generated and used to induce apoptosis of a glioma cell line SHG44. First, the Transwell assay was used to verify that EPCs could home to glioma cells. Then, the co-culture system of TRAIL-overexpressing EPCs and SHG44 cells was established, and the apoptotic state of SHG44 cells 
was examined. Interestingly, the apoptosis percentage and change in protein levels of caspase- 8 and -3 , as well as poly ADP-ribose polymerase (PARP), in SHG44 cells were found to increase remarkably. Taken together, the study demonstrated that the TRAIL-modified EPCs could be a feasible approach for glioma therapy.

\section{Materials and methods}

Cell cultures. Human glioma cell line, SHG44 cells, were purchased from Cell Bank of the Chinese Academy of Sciences and cultured in Dulbecco's modified Eagle's medium (DMEM)/high-glucose medium (HyClone; GE Healthcare, Logan, UT, USA) with $10 \%$ fetal bovine serum (FBS; Gibco; Thermo Fisher Scientific, Inc., Waltham, MA, USA), $0.5 \mathrm{~mm}$ glutamine, and $100 \mathrm{U} / \mathrm{ml}$ penicillin/streptomycin at $37^{\circ} \mathrm{C}$ in a humidified atmosphere containing $5 \% \mathrm{CO}_{2}$ and $95 \%$ air.

EPCs were extracted from the blood of neonatal Sprague-Dawley rats by the density gradient method, as previously described (25), and cultured in endothelial cell growth medium-2 (EGM-2; Lonza, Group, Ltd., Basel, Switzerland) culture medium with $10 \%$ FBS (Gibco; Thermo Fisher Scientific, Inc.), $0.5 \mathrm{~mm}$ glutamine, and $100 \mathrm{U} / \mathrm{ml}$ penicillin/streptomycin in a $5 \% \mathrm{CO}_{2}$ atmosphere at $37^{\circ} \mathrm{C}$.

Immunocytochemical analysis. EPCs were washed twice with phosphate-buffered saline (PBS) and fixed with $4 \%$ paraformaldehyde at $4^{\circ} \mathrm{C}$ overnight. After removing paraformaldehyde and washing the cells with $\mathrm{PBS}, 3 \% \mathrm{H}_{2} \mathrm{O}_{2}$ was used to inactivate endogenous peroxidase for $10 \mathrm{~min}$. The cells were incubated with $5 \%$ bovine serum albumin (BSA) for $1 \mathrm{~h}$ at room temperature to block the nonspecific binding. Then, the primary antibodies (CD31 and CD34, from Abcam, Cambridge, MA, USA) were added onto cells and incubated at $4^{\circ} \mathrm{C}$ for $12 \mathrm{~h}$. Only PBS with 5\% BSA was used as negative control. The cells were washed with PBS three times to remove unbound antibody and then incubated with secondary antibody conjugated to horseradish peroxidase (HRP; ZSGF-BIO, China) for $30 \mathrm{~min}$ at room temperature. After removing the secondary antibody by washing with PBS, the cells were incubated with 3,3'-diaminobenzidine developer, counterstained with hematoxylin, and observed under a microscope.

Immunofluorescence. EPCs were washed with PBS and fixed with $4 \%$ paraformaldehyde after 3 days of lentiviral transfection. The cells were blocked with 5\% BSA for 30 min and then incubated with primary antibodies (TRAIL, DR4, and DR5; Abcam) at $4^{\circ} \mathrm{C}$ for $12 \mathrm{~h}$. After the cells were washed with PBS, secondary antibodies were used for staining (Alexa 594 conjugated; Thermo Fisher Scientific, Inc.; Alexa 405 conjugated; Wuhan Sanying Biotechnology, Wuhan, China). Cells were observed using laser scanning confocal microscopy (Nikon Corporation, Tokyo, Japan).

Angiogenesis assay. BD Matrigel basement membrane matrix (BD Biosciences, Franklin Lakes, NJ, USA) was melted at $0^{\circ} \mathrm{C}$, implanted in a 24-pore culture plate, and then placed in a $37^{\circ} \mathrm{C}$ incubator for $1 \mathrm{~h}$. EPCs were inoculated on the surface of solidified Matrigel $\left(5 \times 10^{4}\right.$ cells per well) and cultured in a $5 \% \mathrm{CO}_{2}$ atmosphere at $37^{\circ} \mathrm{C}$. The cells were observed under a microscope after $6 \mathrm{~h}$, and images were taken under magnification, $\mathrm{x} 40$ and $\mathrm{x} 100$.

Lentiviral infection. The lentivirus with the TRAIL sequence was delivered to overexpress the TRAIL protein in EPCs. The negative control virus carrying green fluorescent protein (GFP) only was used as a control. Both viruses were constructed and packed by Shanghai GeneChem Co., Ltd., Shanghai, China. EPCs were infected using lentiviruses with a multiplicity of infection of 10 on the second day after the first passage of EPCs.

RNA extraction, cDNA synthesis, and reverse transcription-quantitative polymerase chain reaction $(R T-q P C R)$. After a week of lentiviral infection, total RNA was extracted from EPCs in both the groups using TRIzol reagent (Solarbio, Beijing, China), chloroform, and isopropyl alcohol. Then, $1 \mu \mathrm{g}$ of extracted RNA was reverse transcribed into strand cDNA after removing genomic DNA using a PrimeScript Reverse Transcription Reagent kit with gDNA Eraser (TaKaRa Bio Co., Ltd., Otsu, Japan). Real-time quantitative polymerase chain reaction (PCR) was performed on Roche LightCycler (Roche Applied Science, Rotkreuz, Switzerland) using the same reagent kit with cDNA synthesis for the desired gene. The primers were designed and synthesized by Sangon Biotech as follows:

TRAIL sense, 5'-AACTGGGACCAGAGGAAGAAG CAA-3' and antisense, 5'-ATGCCCACTCCTTGATGATTC CCA-3'; Glyceraldehyde-3-phosphate dehydrogenase sense, 5'-TCCTGCACCACCAACTGCTTAG-3' and antisense, 5'-TCCTGCACCACCAACTGCTTAG-3'.

Co-culture.SHG44 cells were cultured with TRAIL-expressing EPCs as the experimental group, or with EPCs expressing only GFP as the control group. Both groups were in the same culture environment: EGM-2 culture medium was used with $10 \% \mathrm{FBS}$, $0.5 \mathrm{mM}$ glutamine, and $100 \mathrm{U} / \mathrm{ml}$ penicillin/streptomycin in a $5 \% \mathrm{CO}_{2}$ atmosphere at $37^{\circ} \mathrm{C}$.

Transwell migration assay. In vitro cell migration assays were performed using 24-well Transwell chambers (12- $\mu \mathrm{m}$ pores; Corning Inc., Corning, NY, USA ). Nontransfected EPCs and TRAIL-transfected EPCs ( $2 \times 10^{4}$ cells per well) were cultured in the top chamber, whereas SHG44 cells, as a positive group, were cultured in the lower chamber. DMEM medium with $10 \%$ FBS only in the lower chamber was used as the control group. After $24 \mathrm{~h}$ of cultivation, the cells on the upper side were removed and the migrated cells were fixed in $4 \%$ paraformaldehyde, stained with 4',6-diamidino-2-phenylindole (DAPI) solution, and examined using a high-content screening system (HCS; Thermo Fisher Scientific, Inc.).

Propidium iodide and Annexin V assays. The cells were gently washed once with PBS on the third day of co-culture and then stained with Annexin V-kFluor594 (Nanjing KeyGen Biotech Co., Ltd., Nanjing, China) for $15 \mathrm{~min}$ at room temperature away from light. The cells were observed under a fluorescence microscope after replacing the staining solution with PBS. The flow cytometry assay was performed using Annexin V-APC (Nanjing KeyGen Biotech Co., Ltd.) and propidium iodide (PI) (Nanjing KeyGen Biotech Co., Ltd.), whose fluorescence 

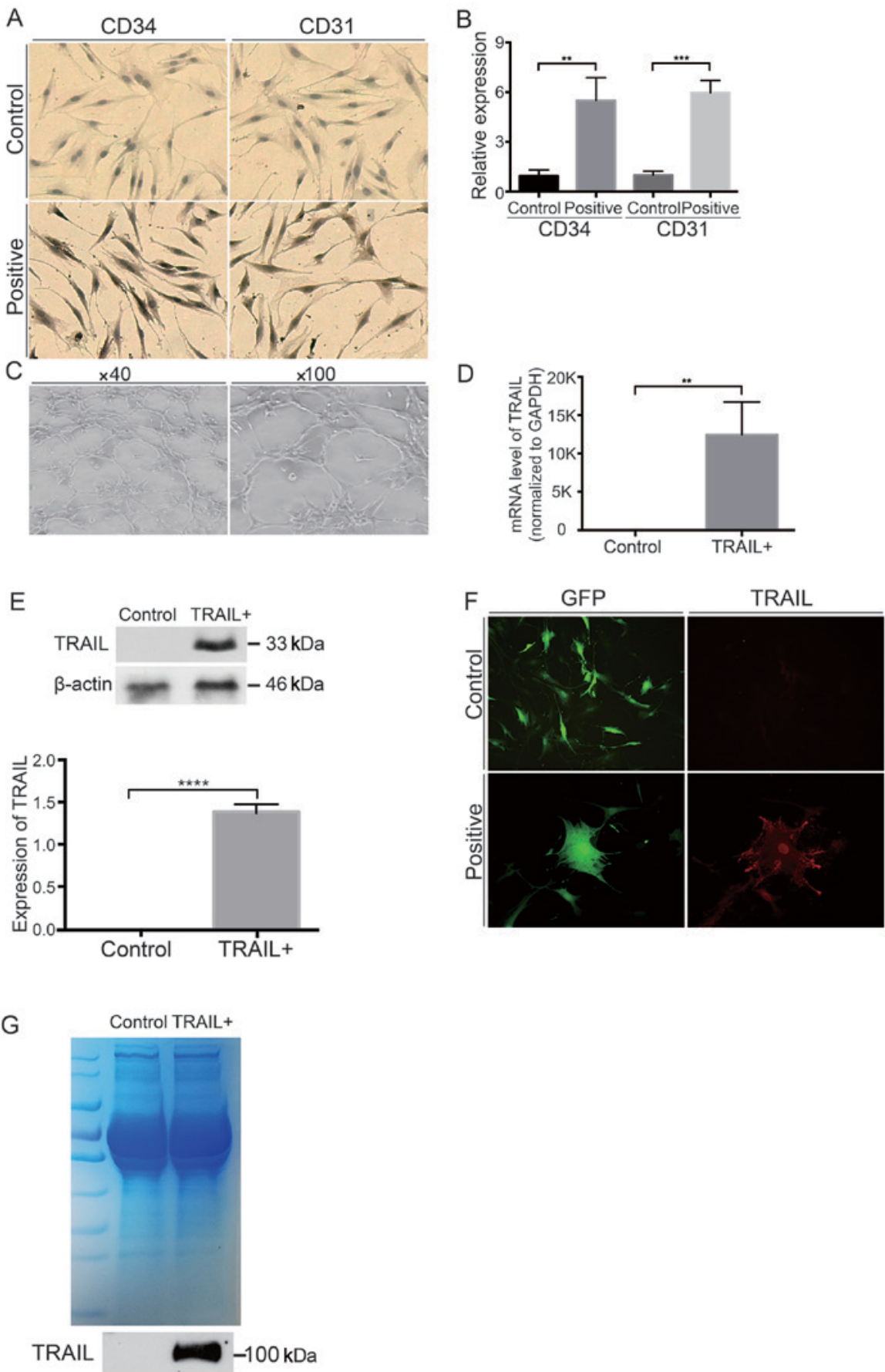

Figure 1. Construction of TRAIL-expressing EPCs. (A and B) Immunocytochemical staining showed that the cells were CD31 and CD34 positive (magnification, x100) ( $\mathrm{P}<0.05$, compared with the control group). (C) EPCs formed capillary-like networks after being seeded onto the Matrigel surface for $6 \mathrm{~h}$. (D and E) EPCs were infected with lentiviruses encoding GFP or TRAIL. The mRNA and protein levels of TRAIL in EPCs were determined using qPCR (D) ( $\mathrm{P}<0.05$, compared with the control group) and western blot analysis. $\beta$-Actin was used as a loading control (E, $\mathrm{P}<0.05$, compared with the control group). (F) EPCs harboring GFP showed green fluorescence, and EPCs harboring TRAIL showed red fluorescence (magnification, x200). (G) The sTRAIL was detected using western blot and Coomassie blue staining. Data are presented as means \pm SD. Three individual experiments were performed for each group. ${ }^{* *} \mathrm{P}<0.01,{ }^{* * *} \mathrm{P}<0.0001$ and ${ }^{* * * * *} \mathrm{P}<0.001$, compared with the control group. TRAIL, tumor necrosis factor-related apoptosis-inducing ligand; EPCs, endothelial progenitor cells; GFP, green fluorescent protein qPCR, quantitative polymerase chain reaction; sTRAIL, soluble extracellular TRAIL; SD, standard deviation.

signals were excited at 633 and $488 \mathrm{~nm}$ and collected at 660 and $610 \mathrm{~nm}$, respectively, to determine the degree of apoptosis of SHG44 cells further. More than 10,000 cells per sample group were collected and divided into EPCs and SHG44 cells on the basis of GFP fluorescence intensity. The percentage of Annexin V-positive SHG44 cells was calculated as an indicator of apoptosis.
Co-immunoprecipitation. The cells from co-culture were homogenized in cell lysis buffer (Solarbio), supplemented with $1 \mathrm{mM}$ phenylmethanesulfonyl fluoride (PMSF) and complete protease inhibitor mixture (Solarbio). The homogenate was centrifuged at $11,000 \mathrm{rpm}$ for $15 \mathrm{~min}$ at $4^{\circ} \mathrm{C}$. The supernatant was incubated with anti-TRAIL (Abcam) crosslinked beads at $4^{\circ} \mathrm{C}$ overnight with rotation. The Pierce Crosslink Magnetic 

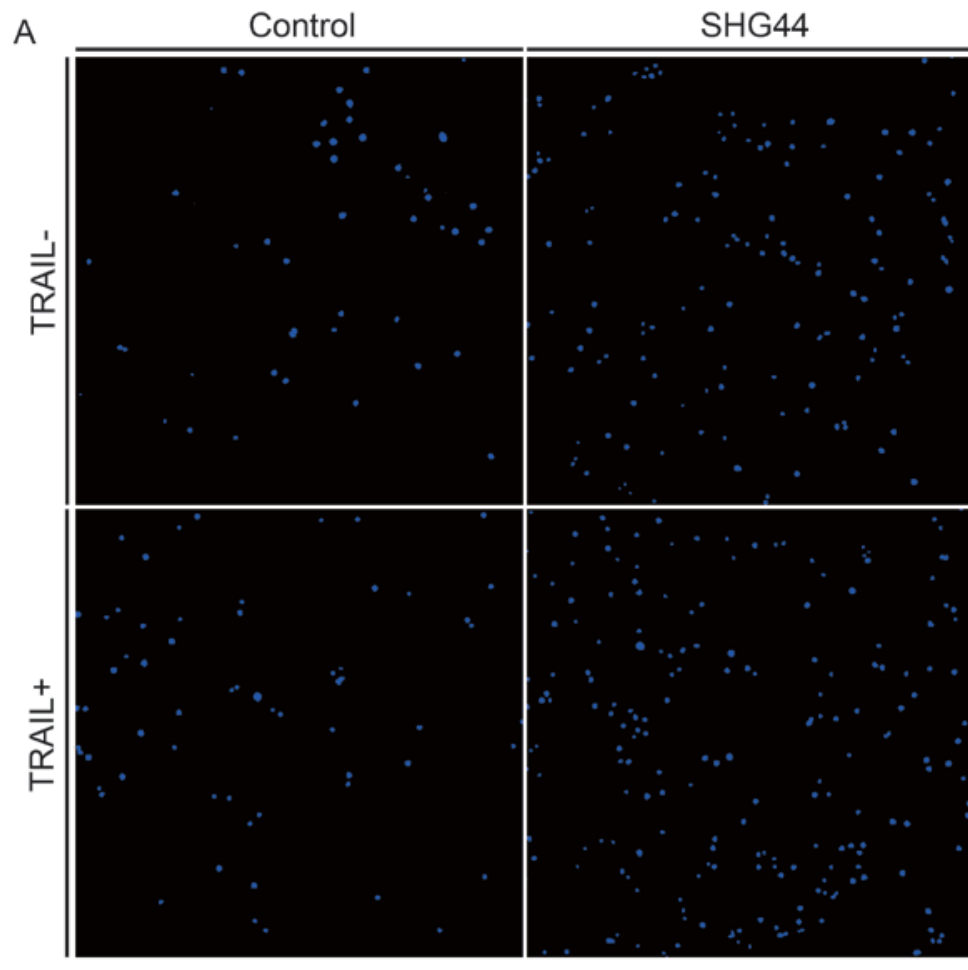

B

C
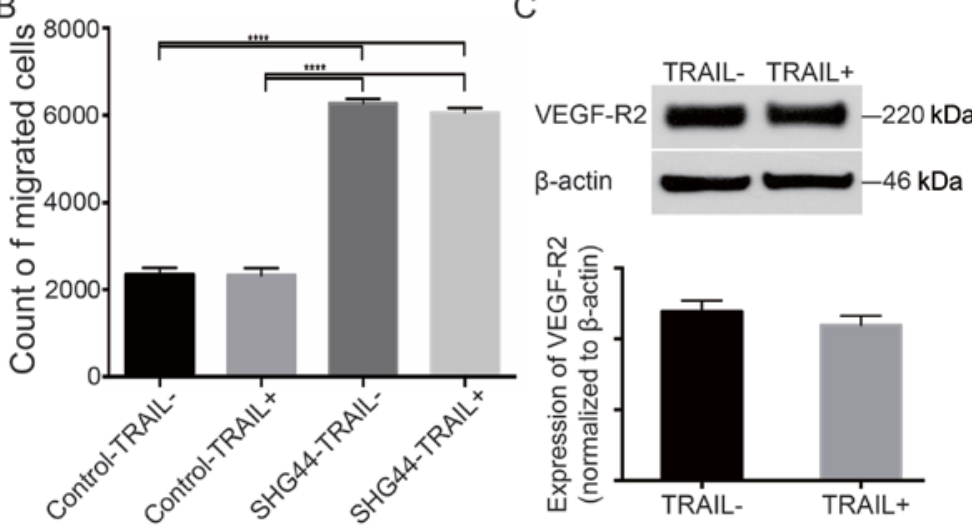

Figure 2. Transwell migration assay of EPCs. (A) Migrated EPCs were stained using DAPI, and images were obtained using HCS of 25 continuous visual fields. (B) Numbers of migrated cells were counted using HCS. The migrated EPCs increased obviously when SHG44 cells were cultured in the lower chamber (magnification, $\mathrm{x} 100)(\mathrm{P}<0.05$, compared with the control group). No significant difference was found between EPCs with or without the overexpression of TRAIL (P>0.05). (C) Expression of VEGF-R2 on control and TRAIL + EPCs. $\beta$-Actin was used as a loading control. Data are presented as means \pm SD. Three individual experiments were performed for each group. ${ }^{* * * * *} \mathrm{P}<0.0001$, compared with the control group. EPCs, endothelial progenitor cells; DAPI, 4',6-diamidino-2-phenylindole, HCS, high-content screening; SD, standard deviation.

IP kit instruction was used for the pretreatment of beads and immunoprecipitation. The associated proteins were detected using western blot analysis. Homogenates from co-culture cells were used as positive controls.

Quantitative immunoblot analysis. The cells in the two groups were collected on third and fifth days of co-culture, homogenized in $1 \mathrm{X}$ cell lysis buffer (Solarbio) supplemented with $1 \mathrm{mM}$ PMSF and complete protease inhibitor mixture (Solarbio) for $30 \mathrm{~min}$ on ice, and then centrifuged at $11,000 \mathrm{rpm}$ for $10 \mathrm{~min}$ at $4^{\circ} \mathrm{C}$. The supernatant was measured using a Bicinchoninic Acid Protein Assay kit (Solarbio). The measurement samples were combined with 5X SDS loading buffer and boiled at $100^{\circ} \mathrm{C}$ for $10 \mathrm{~min}$. For each protein, equal amounts of samples (20-100 $\mu \mathrm{g})$ from each group were analyzed using sodium dodecyl sulfate-polyacrylamide gel electrophoresis as previously described (26). After proteins were transferred onto a polyvinylidene difluoride membrane, the membrane was incubated with 5\% BSA at room temperature for $2 \mathrm{~h}$ to block the nonspecific protein site and then corresponded with primary antibodies [TRAIL from Abcam; DR4 and DR5 from Abcam; vascular endothelial growth factor receptor 2 (VEGF-R2), caspase-8 and -3 , and PARP from Cell Signaling Technology, Inc. (Danvers, MA, USA); $\beta$-actin from ZSGF-BIO] at $4^{\circ} \mathrm{C}$ overnight. This step was followed by incubation with HRP-conjugated secondary antibodies (ZSGF-BIO). Visualization was achieved using a SuperSignal West Pico Trial kit (Thermo Fisher Scientific, Inc.).

Statistical analysis. Data were expressed as mean \pm standard deviation (SD). One-way analysis of variance was used for comparisons among multiple groups, followed by the Student 


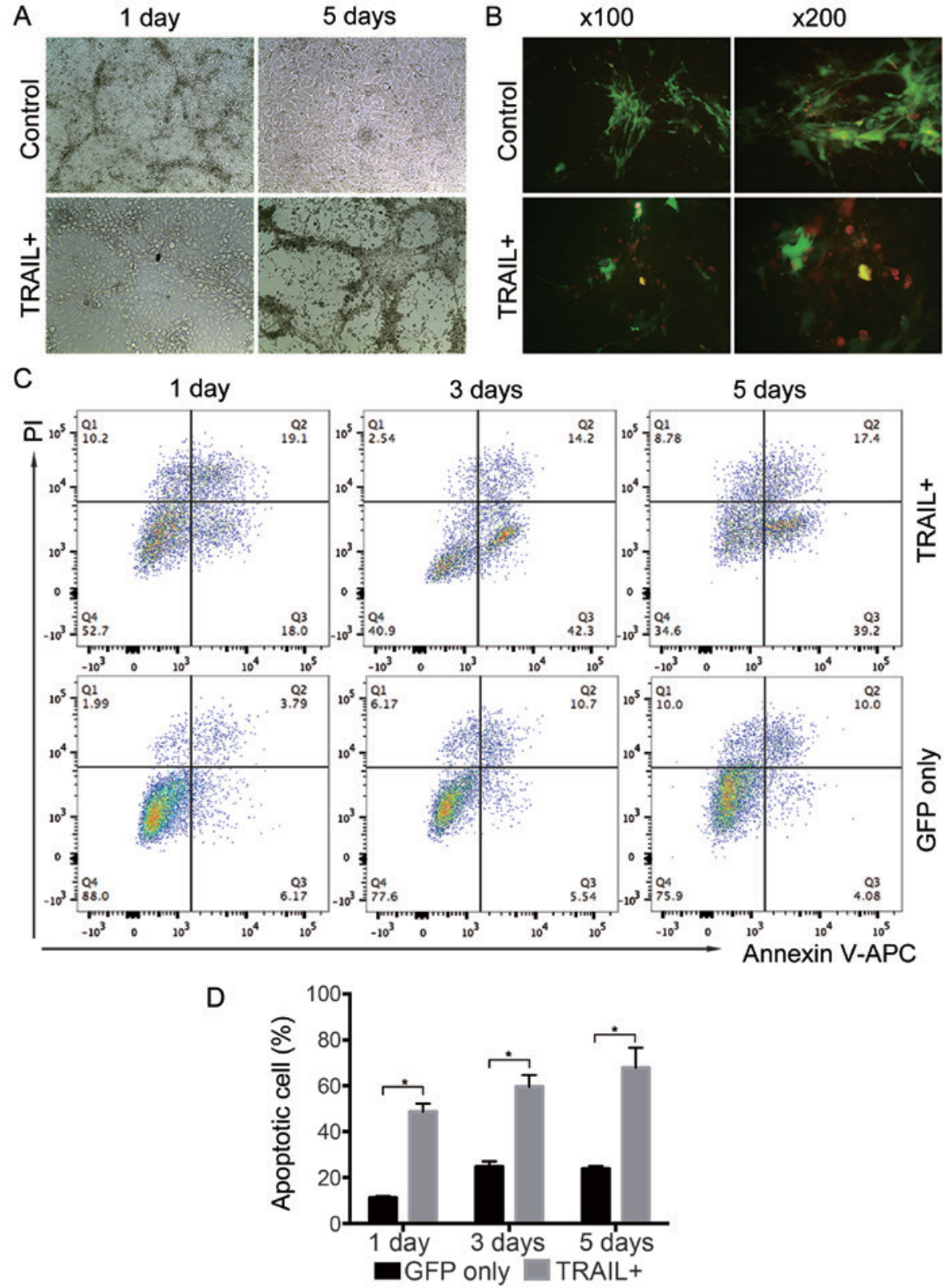

Figure 3. Apoptosis of SHG44 cells co-cultured with EPCs. (A) Cellular growth in the two groups on the first and fifth days after co-culture (magnification, x100). (B) Co-cultured cells were stained with Annexin V-kFluor594 on the fifth day. Apoptotic cells showed red fluorescence, and GFP-harboring EPCs showed green fluorescence. (C and D) The apoptotic rate of SHG44 cells was higher in the TRAIL-positive group than in the control group $(\mathrm{P}<0.05)$. Data are presented as means $\pm \mathrm{SD}$. Three individual experiments were performed for each group. $\mathrm{P}<0.05$, compared with the control group. EPCs, endothelial progenitor cells; GFP, green fluorescent protein; TRAIL, tumor necrosis factor-related apoptosis-inducing ligand; PI, propidium iodide; SD, standard deviation.

post hoc two-tailed test. The unpaired Student t-test was performed for comparisons between the means of two groups. GraphPad 6.0 (GraphPad Software, Inc., La Jolla, CA, USA) was used for all the statistical analyses. $\mathrm{P}<0.05$ was considered to indicate a statistically significant difference.

\section{Results}

Generation of TRAIL-expressing EPCs. Blood cells were extracted from neonatal Sprague-Dawley rats, and the specific surface markers CD31 and CD34 were detected by immunohistochemical analysis (27). Most cells revealed positive reactions to CD31 and CD34 (Fig. 1A and B). The angiogenic ability of EPCs was also identified. The cells inoculated on the Matrigel surface formed tube-like structures within $6 \mathrm{~h}$, as shown in representative images (Fig. 1C). Thus, EPCs were isolated successfully from the blood of neonatal rats.

The isolated EPCs were infected and screened using lentivirus encoding TRAIL. Then, the expression level of TRAIL on EPCs was detected. Total mRNA was also extracted from both TRAIL-transfected EPCs and negative controls. Quantitative PCR was performed to quantify the mRNA levels. The TRAIL mRNA level in infected EPCs significantly increased compared with the control group $(\mathrm{P}<0.05)$ (Fig. 1D). Furthermore, the TRAIL protein level was also enhanced, as determined by western blot analysis $(\mathrm{P}<0.05)$ (Fig. 1E). The location of TRAIL was determined by immunofluorescence staining. TRAIL was found to be mainly expressed on the cell surface and partly in the cytoplasm and nucleus (Fig. 1F). The soluble extracellular TRAIL (sTRAIL) was also detected by 
A
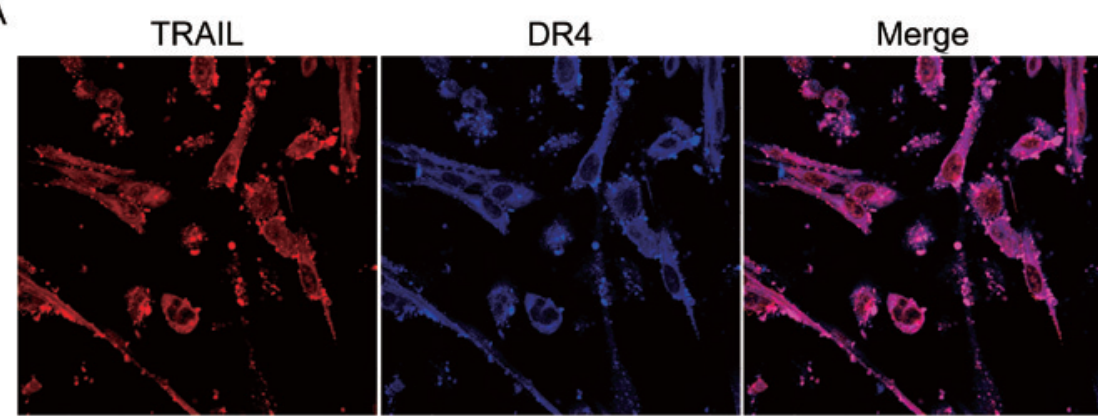

TRAIL

DR4
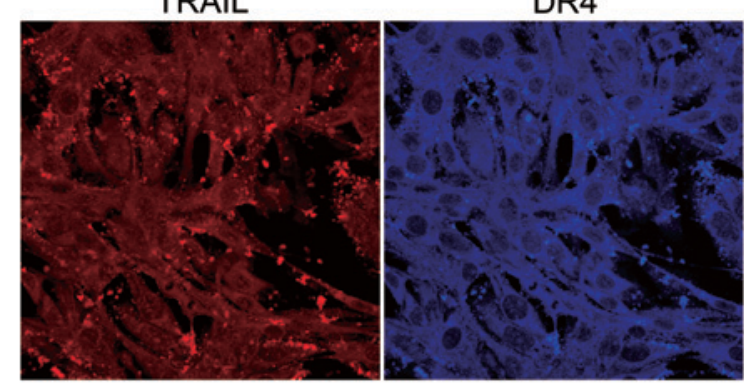

Merge

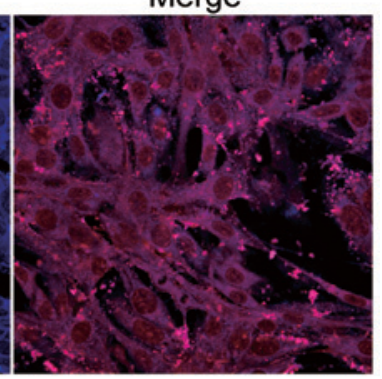

B

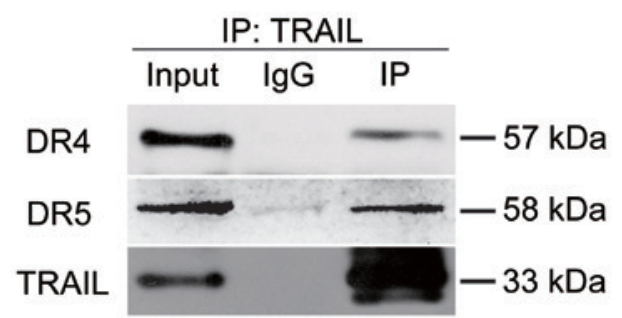

Figure 4. TRAIL combined with DR4 and DR5 on glioma cells. (A) Immunofluorescent co-localization of TRAIL and DR4/5. TRAIL was stained using Alexa 594-conjugated antibody, whereas DR4/5 was stained using Alexa 405-conjugated antibody (magnification, x400). (B) Western blots demonstrated that TRAIL was co-immunoprecipitated with DR4/5. DR4/5, death receptor 4/5; TRAIL, tumor necrosis factor-related apoptosis-inducing ligand.

western blot analysis. It was found that the STRAIL existed in the trimeric form in the culture medium of the TRAIL-positive group (Fig. 1G). These results showed that TRAIL was overexpressed in the EPCs after lentiviral transfection, and the overexpressed TRAIL was further distributed both on the cell membrane and in the culture medium.

Overexpression of TRAIL did not affect the migration of EPCs toward SHG44 cells. A Transwell migration assay was performed to assess the directional migration of EPCs. The number of migrated cells increased significantly when SHG44 cells were cultured in the lower chamber, as evident from statistics and HCS of the images $(\mathrm{P}<0.05)$ (Fig. 2A and $\mathrm{B}$ ). However, similar numbers of the migrated cells were found in the TRAIL-transfected EPCs and controls, indicating that the overexpression of TRAIL did not affect the homing of EPCs to SHG44 cells ( $\mathrm{P}>0.05)$ (Fig. 2A and B). Furthermore, the overexpression of TRAIL did not alter the protein levels of VEGF-R2 in the TRAIL-transfected EPCs compared with the controls ( $\mathrm{P}>0.05)$ (Fig. 2C). These findings indicated that EPCs with the overexpression of TRAIL had the similar ability for directional migration and angiogenesis, which could be used in the following experiments.

Apoptosis of SHG44 in co-culture system with EPCs. A co-culture system in which SHG44 cells were co-cultured with EPCs harboring TRAIL or GFP was established to determine the effect of TRAIL-overexpressing EPCs on SHG44 cells. After 5 days of continuous observation, it was found that the number of SHG44 cells in the TRAIL-expressing EPC-treated group obviously reduced compared with that in the GFP-harboring EPCs (Fig. 3A). Annexin V-kFluor594 was used on the fifth day of co-culture to detect cell apoptosis. More Annexin V-kFluor594-positive cells (red fluorescence) were found around the TRAIL-expressing EPCs (green fluorescence) (Fig. 3B) in the TRAIL-expressing EPC-treated group compared with the GFP group. It indicated that the TRAIL-expressing EPCs could induce SHG44 cell apoptosis. Then, the flow cytometry assay was used to measure the apoptotic rate of SHG44 cells. The cells were collected and stained with Annexin V-APC/PI on the first, third, and fifth days after co-culturing. The apoptotic rate of SHG44 cells significantly increased by co-culturing with the TRAIL-expressing EPCs, compared with that of the cells harboring GFP, after separating EPCs and SHG44 cells based on GFP fluorescence intensity, (Fig. 3C and D). These findings indicated that TRAIL-positive EPCs could increase SHG44 cell apoptosis.

TRAIL bound with DR4/5 on glioma cells. The study demonstrated that TRAIL-positive EPCs could induce SHG44 cell apoptosis obviously. Next, immunofluorescent 
A
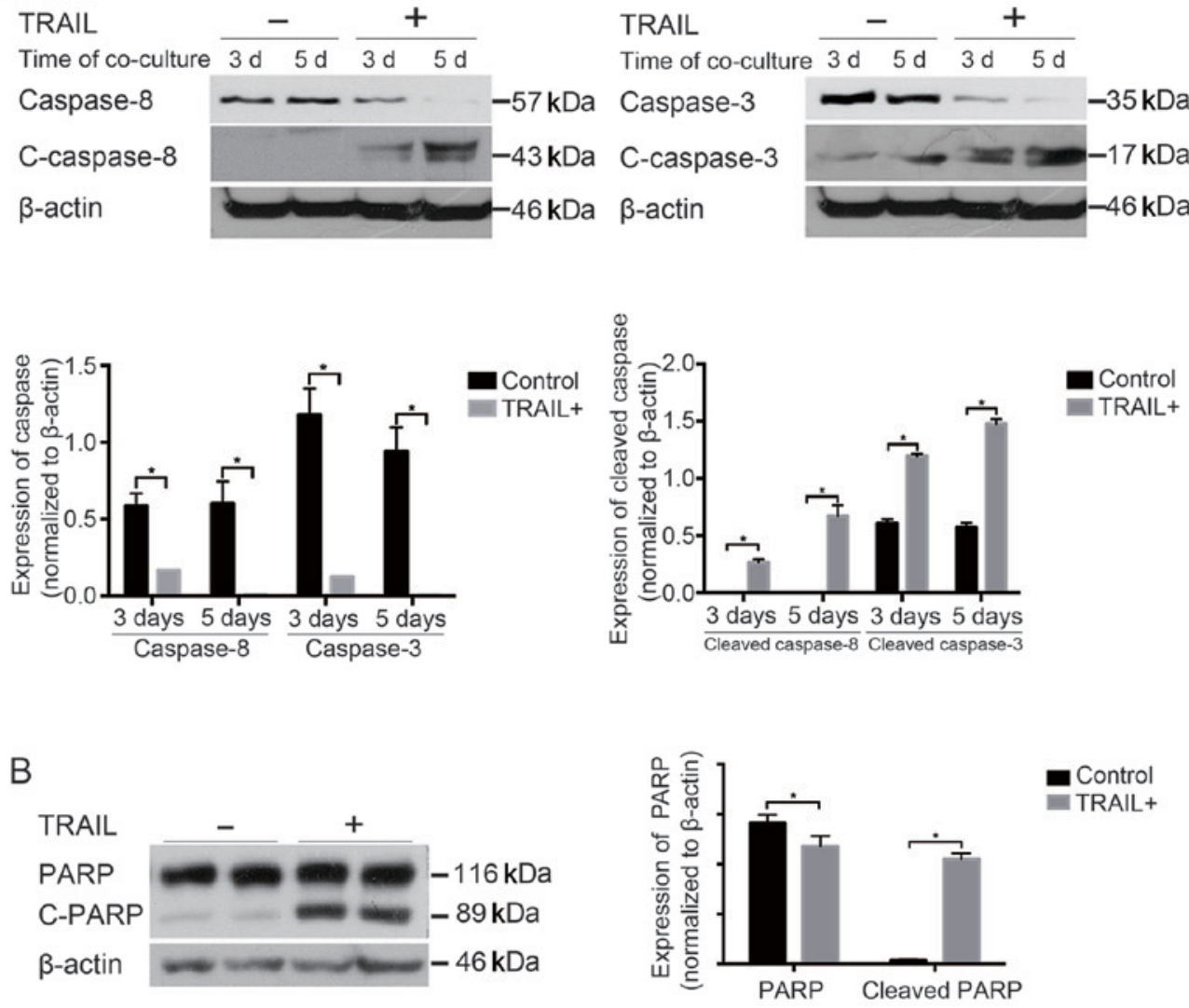

Figure 5. TRAIL enhanced the activation of caspases and PARP. (A) TRAIL expression induced the activation of caspase- 8 and -3 significantly. $\beta$-Actin was used as a loading control $(\mathrm{P}<0.05)$. (B) The cleaved PARP increased obviously in the TRAIL-positive group, whereas the original PARP showed a mild decrease. $\beta$-Actin was used as a loading control $(\mathrm{P}<0.05)$. Data are presented as means \pm SD. Three individual experiments were performed for each group. ${ }^{*} \mathrm{P}<0.05$, compared with the control group. PARP, poly ADP-ribose polymerase; TRAIL, tumor necrosis factor-related apoptosis-inducing ligand; SD, standard deviation.
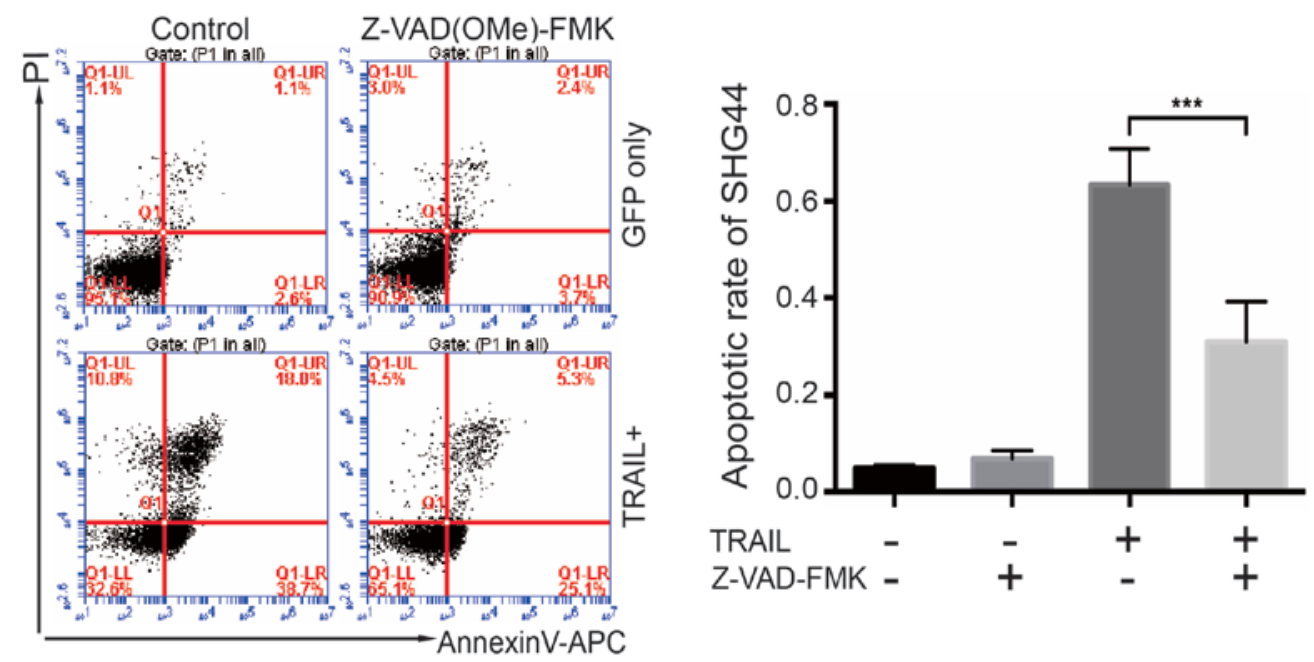

Figure 6. Apoptosis in the co-culture system could be reversed using a caspase inhibitor. The apoptotic rate significantly reduced with Z-VAD(OMe)-FMK $(\mathrm{P}<0.05)$. Data are presented as means $\pm \mathrm{SD}$. Three individual experiments were performed for each group. ${ }^{* * *} \mathrm{P}<0.001$, compared with the control group. $\mathrm{PI}$, propidium iodide; TRAIL, tumor necrosis factor-related apoptosis-inducing ligand; SD, standard deviation.

co-localization and co-immunoprecipitation were used to verify the interaction of TRAIL and death receptor so as to explore the apoptotic mechanism further. Double-channel confocal imaging revealed that TRAIL showed red fluorescence, TRAIL co-localized with DR4 and DR5 (DR4 and DR5) showed blue fluorescence (Fig. 4A). The total proteins from the co-culture system were also extracted to test the interaction between TRAIL and DR4/5 by immunoblotting. It was found that TRAIL co-immunoprecipitated with DR4/5 in SHG44 cells (Fig. 4B). 
TRAIL enhanced the activation of caspases and PARP. Next, the expression levels of pro/cleaved caspase-8, pro/caspase-3, and PARP were examined. The expression levels of cleaved caspase- 8 and -3 significantly increased and were further enhanced with time in the co-culture system, whereas the expression levels of pro-caspase- 8 and -3 decreased significantly, compared with the GFP group $(\mathrm{P}<0.05)$ (Fig. 5A). Similar changes were observed in the cleaved PARP. In contrast, the amount of full-length PARP was much less in the TRAIL-positive group than in the GFP group $(\mathrm{P}<0.05)$ (Fig. 5B), indicating that PARP was largely activated under the effect of TRAIL on EPCs.

Apoptosis in the co-culture system could be reversed by a caspase inhibitor. The caspase inhibitor Z-VAD(OMe)-FMK was used for 5 days of co-culture, and the apoptosis of SHG44 cells was detected to verify the involvement of TRAIL-expressing EPCs further. Fig. 6 shows that the apoptotic rate was significantly reversed when Z-VAD(OMe)-FMK was added at the concentration of $20 \mu \mathrm{mol} / \mathrm{l}$ (Fig. 6).

\section{Discussion}

Gliomas, the most common malignant tumors in CNS, exhibit highly aggressive behavior. The therapeutic effect on glioma is still not improved drastically despite various new therapies reported for this tumor. In this study, the extracted EPCs from the blood of neonatal Sprague-Dawley rats were identified by detecting specific surface markers CD31 and CD34 as well as the capability of vasculogenesis. TRAIL was found to increase the apoptosis of SHG44 cells significantly. This trend could be reversed by caspase-8 inhibitor. The mechanism underlying this phenomenon might be related to the increased pro-apoptotic protein levels of cleaved caspase-3, caspase- 8 , and PARP.

EPCs can self-proliferate and differentiate into the endothelial lineage and express CD31, CD34, CD133, and VEGFR-2 on their surface $(28,29)$. They have the capability of vasculogenesis and are involved in tumor vessel neogenesis. Many studies investigated the relationship between EPCs and tumors (22). Most previous studies focused on anti-angiogenic treatment by suppressing the mobilization and homing of EPCs to tumors (20). Only a few studies used EPCs as a vector for treatment (21). EPCs harboring specific matrix metallopeptidase-12 (MMP-12) could inhibit melanoma growth (30). On the contrary, EPCs releasing CD40 ligand could induce the increase in cleaved caspase- 3 and -7 in metastatic breast cancer (31). These findings proved that EPCs could be used for tumor treatment. Growing evidence indicated that EPCs could move across the BBB and home to brain tumors (23). EPCs can serve as a medium for anti-glioma therapy. The Transwell assay indicated that EPCs had the capability of homing to glioma cells, as previously reported. The present study also proved that lentiviral infection had little influence on the migration ability of EPCs. Therefore, it was feasible to use the TRAIL-expressing EPCs as a therapeutic vector after lentiviral infection.

TRAIL induces apoptosis through binding to DR4 or DR5 on the surface of cells, recruiting and activating caspase- 8 via the death domains of DRs and adaptor protein Fas-associated death domain (FADD) $(9,32)$. Activated caspase- 8 cleaves pro-caspase-3, inducing cell apoptosis $(33,34)$. The low bioavailability of TRAIL limits its application despite its immense potential for anti-tumor therapy. Attempts were made to address this limitation through enhancing the stability of TRAIL (35-38) and concentrating TRAIL in the tumor site (39-42). In this study, EPCs were used as a vector for TRAIL, continuously producing TRAIL besides tumor cells. The findings indicated that TRAIL delivered by EPCs could bind to DR4 or DR5 on the surface of glioma cells. Correspondingly, the activated caspase- 8 and -3 , and cleaved PARP increased obviously. In other words, TRAIL-expressing EPCs could promote the apoptosis of SHG44 cells through activating the death receptor pathway.

In conclusion, the present study demonstrated that TRAIL-overexpressing EPCs could migrate toward SHG44 cells and induce apoptosis by activating the death receptor pathway. The findings suggested that TRAIL and EPCs could be combined for glioma therapy.

\section{Acknowledgements}

The present study is supported by the National Natural Science Foundation of China (grant no. 81100928) and the Henan Medical Science Research Project (grant no. 2011020010). The present study was supported by grants from the National Natural Science Fund of China (no. 342600531547), the National Natural Science Foundation of China (nos. 81270270 and 81470524, for WZ), PhD Educational Award from the Ministry of Education (no. 20134101110013, for WZ), and the national Key R\&D Program for 'Key Projects' from the Ministry of Science and Technology (no. 2016YFA0501800, for WZ).

\section{References}

1. Ostrom QT, Gittleman H, Fulop J, Liu M, Blanda R, Kromer C, Wolinsky Y, Kruchko C and Barnholtz-Sloan JS: CBTRUS statistical report: Primary brain and central nervous system tumors diagnosed in the United States in 2008-2012. Neuro Oncol 4 (17 Suppl): iv1-iv62, 2015.

2. Wang X, Chen JX, Zhou Q, Liu YH, Mao Q, You C, Chen N, Xiong L, Duan J and Liu L: Statistical report of central nervous system tumors histologically diagnosed in the Sichuan province of China from 2008 to 2013: A West China Glioma Center report. Ann Surg Oncol 23 (Suppl 5): S946-S953, 2016.

3. Lim B, Allen JE, Prabhu VV, Talekar MK, Finnberg NK and El-Deiry WS: Targeting TRAIL in the treatment of cancer: New developments. Expert Opin Ther Targets 19: 1171-1185, 2015.

4. Kichev A, Rousset CI, Baburamani AA, Levison SW, Wood TL, Gressens P, Thornton C and Hagberg H: Tumor necrosis factor-related apoptosis-inducing ligand (TRAIL) signaling and cell death in the immature central nervous system after hypoxia-ischemia and inflammation. J Biol Chem 289: 9430-9439, 2014.

5. Bo Y, Guo G and Yao W: MiRNA-mediated tumor specific delivery of TRAIL reduced glioma growth. J Neurooncol 112: 27-37, 2013 .

6. Förster A, Falcone FH, Gibbs BF, Preussner LM, Fiebig BS, Altunok H, Seeger JM, Cerny-Reiterer S, Rabenhorst A, Papenfuss K, et al: Anti-Fas/CD95 and tumor necrosis factor-related apoptosis-inducing ligand (TRAIL) differentially regulate apoptosis in normal and neoplastic human basophils. Leuk Lymphoma 54: 835-842, 2013.

7. Guo L, Fan L, Pang Z, Ren J, Ren Y, Li J, Chen J, Wen Z and Jiang X: TRAIL and doxorubicin combination enhances anti-glioblastoma effect based on passive tumor targeting of liposomes. J Control Release 154: 93-102, 2011.

8. Gao JQ, Lv Q, Li LM, Tang XJ, Li FZ, Hu YL and Han M: Glioma targeting and blood-brain barrier penetration by dual-targeting doxorubincin liposomes. Biomaterials 34: 5628-5639, 2013. 
9. Holoch PA and Griffith TS: TNF-related apoptosis-inducing ligand (TRAIL): A new path to anti-cancer therapies. Eur J Pharmacol 625: 63-72, 2009.

10. Kelley SK and Ashkenazi A: Targeting death receptors in cancer with Apo2L/TRAIL. Curr Opin Pharmacol 4: 333-339, 2004.

11. Bagci-Onder T, Agarwal A, Flusberg D, Wanningen S, Sorger P and Shah K: Real-time imaging of the dynamics of death receptors and therapeutics that overcome TRAIL resistance in tumors. Oncogene 32: 2818-2827, 2013.

12. Perlstein B, Finniss SA, Miller C, Okhrimenko H, Kazimirsky G, Cazacu S, Lee HK, Lemke N, Brodie S, Umansky F, et al: TRAIL conjugated to nanoparticles exhibits increased anti-tumor activities in glioma cells and glioma stem cells in vitro and in vivo. Neuro Oncol 15: 29-40, 2013.

13. Redjal N, Zhu Y and Shah K: Combination of systemic chemotherapy with local stem cell delivered S-TRAIL in resected brain tumors. Stem Cells 33: 101-110, 2015.

14. Balyasnikova IV, Prasol MS, Ferguson SD, Han Y, Ahmed AU, Gutova M, Tobias AL, Mustafi D, Rincón E, Zhang L, et al: Intranasal delivery of mesenchymal stem cells significantly extends survival of irradiated mice with experimental brain tumors. Mol Ther 22: 140-148, 2014.

15. Choi SA, Yun JW, Joo KM, Lee JY, Kwak PA, Lee YE, You JR, Kwon E, Kim WH, Wang KC, et al: Preclinical biosafety evaluation of genetically modified human adipose tissue-derived mesenchymal stem cells for clinical applications to brainstem glioma. Stem Cells Dev 25: 897-908, 2016.

16. Asahara $\mathrm{T}$ and Kawamoto A: Endothelial progenitor cells for postnatal vasculogenesis. Am J Physiol Cell Physiol 287: C572-C579, 2004.

17. Asahara T, Murohara T, Sullivan A, Silver M, van der Zee R, Li T, Witzenbichler B, Schatteman G and Isner JM: Isolation of putative progenitor endothelial cells for angiogenesis. Science 275: 964-967, 1997

18. Fang J, Chen X, Wang S, Xie T, Du X, Liu H, Wang S, Li X, Chen J, Zhang B, et al: The expression of $\mathrm{P} 2 \mathrm{X}_{7}$ receptors in EPCs and their potential role in the targeting of EPCs to brain gliomas. Cancer Biol Ther 16: 498-510, 2015.

19. Varma NR, Shankar A, Iskander A, Janic B, Borin TF, Ali MM and Arbab AS: Differential biodistribution of intravenously administered endothelial progenitor and cytotoxic T-cells in rat bearing orthotopic human glioma. BMC Med Imaging 13: 17, 2013.

20. Flamini V, Jiang WG, Lane J and Cui YX: Significance and therapeutic implications of endothelial progenitor cells in angiogenic-mediated tumour metastasis. Crit Rev Oncol Hematol 100: 177-189, 2016.

21. Marçola M and Rodrigues CE: Endothelial progenitor cells in tumor angiogenesis: Another brick in the wall. Stem Cells Int 2015: 832649, 2015.

22. de la Puente P, Muz B, Azab F and Azab AK: Cell trafficking of endothelial progenitor cells in tumor progression. Clin Cancer Res 19: 3360-3368, 2013.

23. Wang L, Chen L, Wang Q, Wang L, Wang H, Shen Y, Li X, $\mathrm{Fu}$ Y, Shen Y and Yu Y: Circulating endothelial progenitor cells are involved in VEGFR-2-related endothelial differentiation in glioma. Oncol Rep 32: 2007-2014, 2014.

24. Zhang SH, Xiang P, Wang HY, Lu YY, Luo YL and Jiang H: The characteristics of bone marrow-derived endothelial progenitor cells and their effect on glioma. Cancer Cell Int 12: 32, 2012.

25. Kang SD, Carlon TA, Jantzen AE, Lin FH, Ley MM, Allen JD, Stabler TV, Haley NR, Truskey GA and Achneck HE: Isolation of functional human endothelial cells from small volumes of umbilical cord blood. Ann Biomed Eng 41: 2181-2192, 2013.

26. Zhao W, Waggoner J, Zhang ZG, Lam CK, Han P, Qian J, Schroder PM, Mitton B, Kontrogianni-Konstantopoulos A, Robia SL and Kranias EG: The anti-apoptotic protein HAX-1 is a regulator of cardiac function. Proc Natl Acad Sci USA 106: 20776-20781, 2009.

27. Huang G, Tao L, Shen S and Chen L: Hypoxia induced CCL28 promotes angiogenesis in lung adenocarcinoma by targeting CCR3 on endothelial cells. Sci Rep 6: 27152, 2016.
28. Peichev M, Naiyer AJ, Pereira D, Zhu Z, Lane WJ, Williams M, Oz MC, Hicklin DJ, Witte L, Moore MA and Rafii S: Expression of VEGFR-2 and AC133 by circulating human CD34(+) cells identifies a population of functional endothelial precursors. Blood 95: 952-958, 2000.

29. Fadini GP, Losordo D and Dimmeler S: Critical reevaluation of endothelial progenitor cell phenotypes for therapeutic and diagnostic use. Circ Res 110: 624-637, 2012.

30. Laurenzana A, Biagioni A, D'Alessio S, Bianchini F, Chillà $\mathrm{A}$ Margheri F, Luciani C, Mazzanti B, Pimpinelli N, Torre E, et al: Melanoma cell therapy: Endothelial progenitor cells as shuttle of the MMP12 uPAR-degrading enzyme. Oncotarget 5: 3711-3727, 2014.

31. Purwanti YI, Chen C, Lam DH, Wu C, Zeng J, Fan W and Wang S: Antitumor effects of CD40 ligand-expressing endothelial progenitor cells derived from human induced pluripotent stem cells in a metastatic breast cancer model. Stem Cells Transl Med 3: 923-935, 2014.

32. de Miguel D, Lemke J, Anel A, Walczak H and Martinez-Lostao L: Onto better TRAILs for cancer treatment. Cell Death Differ 23: 733-747, 2016.

33. Ozören N and El-Deiry WS: Defining characteristics of Types I and II apoptotic cells in response to TRAIL. Neoplasia 4: 551-557, 2002.

34. Rudner J, Jendrossek V, Lauber K, Daniel PT, Wesselborg S and Belka C: Type I and type II reactions in TRAIL-induced apoptosis-results from dose-response studies. Oncogene 24: 130-140, 2005.

35. Rozanov DV, Savinov AY, Golubkov VS, Rozanova OL, Postnova TI, Sergienko EA, Vasile S, Aleshin AE, Rega MF, Pellecchia M and Strongin AY: Engineering a leucine zipper-TRAIL homotrimer with improved cytotoxicity in tumor cells. Mol Cancer Ther 8: 1515-1525, 2009.

36. Berg D, Lehne M, Müller N, Siegmund D, Münkel S, Sebald W, Pfizenmaier K and Wajant H: Enforced covalent trimerization increases the activity of the TNF ligand family members TRAIL and CD95L. Cell Death Differ 14: 2021-2034, 2007.

37. Schneider B, Münkel S, Krippner-Heidenreich A, Grunwald I, Wels WS, Wajant H, Pfizenmaier K and Gerspach J: Potent antitumoral activity of TRAIL through generation of tumor-targeted single-chain fusion proteins. Cell Death Dis 1: e68, 2010

38. Siegemund M, Pollak N, Seifert O, Wahl K, Hanak K, Vogel A, Nussler AK, Göttsch D, Münkel S, Bantel H, et al: Superior antitumoral activity of dimerized targeted single-chain TRAIL fusion proteins under retention of tumor selectivity. Cell Death Dis 3: e295, 2012.

39. Mitchell MJ, Wayne E, Rana K, Schaffer CB and King MR: TRAIL-coated leukocytes that kill cancer cells in the circulation. Proc Natl Acad Sci USA 111: 930-935, 2014.

40. Loi M, Becherini P, Emionite L, Giacomini A, Cossu I, Destefanis E, Brignole C, Di Paolo D, Piaggio F, Perri P, et al: sTRAIL coupled to liposomes improves its pharmacokinetic profile and overcomes neuroblastoma tumour resistance in combination with Bortezomib. J Control Release 192: 157-166, 2014.

41. Yan C, Li S, Li Z, Peng H, Yuan X, Jiang L, Zhang Y, Fan D, $\mathrm{Hu} X$, Yang $\mathrm{M}$ and Xiong D: Human umbilical cord mesenchymal stem cells as vehicles of CD20-specific TRAIL fusion protein delivery: A double-target therapy against non-Hodgkin's lymphoma. Mol Pharm 10: 142-151, 2013.

42. Seifert O, Pollak N, Nusser A, Steiniger F, Rüger R, Pfizenmaier K and Kontermann RE: Immuno-LipoTRAIL: Targeted delivery of TRAIL-functionalized liposomal nanoparticles. Bioconjug Chem 25: 879-887, 2014.

This work is licensed under a Creative Commons Attribution-NonCommercial-NoDerivatives 4.0 International (CC BY-NC-ND 4.0) License. 